\title{
Research on the treatment of
} metastatic colon cancer patients treated by FOLFOXIRI: Efficacy and toxicity of first-line treatment in stage IV metastatic colorectal

\section{cancer patients}

\begin{abstract}
Background: In recent years, the new trend in treatment of colorectal cancer is the use of target drug. We conduct the study to evaluate efficacy of triplet combination chemotherapy of 5-fluorouracil/leucovorin, oxapilatin, and irinotecan (FOLFOXIRI) in the treatment of stage IV colorectal cancer patients.
\end{abstract}

Method: Uncontrolled clinical trial carried on 39 stage IV colorectal cancer patients

Results: The overall response rate of the treatment was $75 \%$. Mean of progression free survival was $13.4 \pm 9$ months. The overall survival rate at 12 th month and 24 th month were $90 \%$ and $76 \%$, respectively. The proportion of granulocytopenia was $48.9 \%$, no grade 3 or 4 . Side effect beyond hematology was most seen in hepatic toxicity with $52.5 \%$, mainly at grade 1 . Vomiting was $18.3 \%$, all at grade 1 . Other toxicity was very low at percentage.

Conclusion: The triplet combination FOLFOXIRIN chemotherapy improves the outcome of patient with metal colorectal cancer in term of response rate, overall survival rate and progression free survival, and the level of toxicity was acceptable..

Keywords: FOLFOXIRI - Stage IV colorectal cancer - Response rate - Progression free survival " Overall survival - Toxicity

Submitted Date: 03 August 2018; Accepted Date: 23 August 2018; Published Date: 7 August 2018

\section{Introduction}

Colorectal cancer is one of the most commonly diagnosed cancers and a leading cause of cancer-related mortality worldwide. Treatment of metastatic colorectal cancer is still difficult, with median overall survival (OS) of 16-25 months and a 5-years survival rate of $11 \%$ [1]. At present, 5 fluorouracilimines, oxaliplatin and irinotecan are still three "backbone" drugs in the treatment of metastatic colorectal cancer.

A meta-analysis presented that the increase of overall survival rate significantly associate with the use of all 5-fluorouracil, oxapilatin and irinotecan over the total duration of treatment [2]. Currently, in accordance with recommendation by many cancer organizations around the world, these three drugs are used step by step through two-drug regimen (FOLFOX/XELOX, FOLFIRI/ XELIRI). However, not all $100 \%$ of patients are treated with all 3 these drugs because some patients abandoned the following treatment lines, or their condition does not allow second-line treatment.
Trinh Le Huy and Bui My Hanh* Hanoi Medical university, Hanoi, Vietnam

*Author for correspondence: E-mail: buimyhanh@hmu.edu.vn 
Thus, the idea of using all three drugs from the first-line treatment was sprouted more than 10 years ago. In 2002, the first two Phase II studies reported the efficacy and safety of combination of Oxapilatin/ Irinotecan/5FULV (FOLFOXIRI) at the first stage [3]. Subsequently, many phase III studies were performed with satisfactory results of this regimen. Since 2010, FOLFOXIRI were recommended in NCCN Clinical Practice Guidelines in Oncology (NCNN Guidelines).

In Vietnam, FOLFOXIRI regimen is gradually being introduced at some oncology facilities due to its efficacy and feasibility. However, since then there was no clinical trial established to evaluate effectiveness as well as toxicity of FOLFOXIRI in Vietnamese patient with metastatic colorectal cancer. Therefore, we carried out the work with the aim to perform an initial review addressing the efficacy and toxicity of treatment with FOLFOXIRI regimen in stage IV metastatic colorectal cancer patients.

\section{Methods}

\section{Study design}

This study was single-arm, uncontrolled prospective clinical trial. All eligible patients will be selected from October 2013 to September 2014 in the Oncology and Palliative Care Department, Hanoi Medical University Hospital. Patients were informed of the investigational nature of the study and provided their writen informed consent before registration onto the study. This study was registered with ClinicalTrials.gov (No. NCT03504618).

\section{Patient selection and study process}

Main eligibility criteria were as followed: histologically confirmed diagnosis of colorectal adenocarcinoma with unresectable metastatic disease; age between 20 and 75 years; Eastern Cooperative Oncology Group (ECOG) performance status (PS) score of 0 or 1; the patients were treated by at least 3 cycles of FOLFOXIRIN in the first-line treatment; liver, kidney and hematological functions before treatment were in normal limits; no systematic cardiac disease, myocardial infarction in the last 24 months or uncontrolled arrhythmia.

General information was collected by questioning patients and their family member to record age, gender, medical history, reasons of hospitalization, signs and symptoms. Pretreatment assessment included history and physical examination, performance status assessment, complete blood cell with differential and platelet counts, complete blood profile, CEA level measurement, colonoscopy, chest X-ray or computed tomography (CT) scan, abdominal CT scan and/ or ultrasound, electrocardiogram and any other appropriate diagnostic procedure to evaluate metastatic sites. During treatment, a physical examination was performed every 2 weeks. Metastatic sites were reevaluated every 8 weeks. Abdominal CT scan or MRI was required in order to evaluate liver and abdominal metastases.

The treatment of FOLFOXIRI regimen at first line with dosage: On Day 1, irinotecan $165 \mathrm{mg} / \mathrm{m} 2$, intravenous infusion for 1 hour, followed immediately by oxaliplatin $85 \mathrm{mg} / \mathrm{m}^{2}$, infused concomitantly for 2 hours, followed immediately by calcium folinate 200 $\mathrm{mg} / \mathrm{m}^{2}$, intravenously for 2 hours, followed immediately by 5 -FU $3200 \mathrm{mg} / \mathrm{m}^{2}$ infused as a 48 hours continuous infusion. On Days 2 and to 4, regular supportive therapy was as follows: oral dexamethasone $4 \mathrm{mg}$ and aprepitant $80 \mathrm{mg}$ on Days 2 and 3; and finally oral dexamethasone $4 \mathrm{mg}$ on Day 4 . Treatment was repeated every 2 weeks. The chemotherapy was continued until evidence of progression, unacceptable adverse event or severity of toxic, or consent withdrawal.

Evaluation of treatment response was made according to RECIST (Response Evaluation Criteria in Solid Tumors) criteria version 1.1 developed by Eisenhauer et al. 2009. The toxicities of FOFOXIRI chemotherapy were observed weekly and graded according to standard National Cancer Institute Common Terminology Criteria for Adverse Events version 4.0. Patients with disease stability or continuous response after 6 cycles were shifted to maintenance phase with oral Capecitabine (Xeloda). During maintenance treatment, patients were re-examined before Capecitabine administration.

\section{Statistical analysis}

All the data collected was analyzed and measured using SPSS 16.0 software. The Kaplan-Meier method was used to estimate progression-free survival (PFS) and overall survival (OS) from the date of treatment start. Chi-square test was used to analyze dichotomous variables and T-student test was used to compare median values. Statistical significance was set at $P$ value $<0.05$.

\section{Result}

A total of 39 patients with unresectable metastatic colorectal carcinoma were recruited to the study. There were 26 males and 13 females, with a median age of 52.9 years (range 25-70). Half of number of participants had taken chemotherapy for their disease 
previously. Abdominal pain was the most common reason for hospitalization, with $74.4 \%$. Majority of patients were with PS-1 (82.1\%), while performance status (PS) score of 0 accounted for percentage of $17.9 \%$. No case of PS-2 or above. Bowel obstruction was the most common symptom (43.6\%), followed by papable abdominal mass of $20.5 \%$ at the time of diagnosis.

Moderately differenciated adecarcinoma was responsible for two third of the study population, followed by participants having type of histopathology as mucinous adenocarcinoma. The peritoneum was more commonly found in patients with mucinous colorectal cancer $(20.5 \%$ compared with $5.1 \%$ of patients with moderately differenciated adenocarcinoma).

Regarding to primary tumor distribution, left-sided colorectal cancer had the highest percentage of $61.5 \%$.
Of which, primary tumors were more frequently distributed at sigmoid colon (41\%) and liver (17.9\%), other locations accounted for proportion of $41.1 \%$. Liver was the most prevalent distant metastatic site (64.1\%), whereas patients with peritoneal metastasis and pulmonary metastasis were $25.6 \%$ and $17.9 \%$, respectively. Retropenitoreal lymp node occurred in $30.8 \%$ of metastatic colorectal patients. $26(66.7 \%)$ patients had CEA levels of $20 \mathrm{ng} / \mathrm{ml}$ or below before treatment (Tables 1 and 2).

All the participating patients had measurable disease and were evaluate for response. Five patients discontinued the chemotherapy prematurely because of disease progression after 3 cycles and geographical distance. 29 patients achieved partial remission and no case of complete response, therefore, the overall response and disease control rates were $75 \%$ and $91.7 \%$,

Table 1: Clinic-pathology features of metastatic colon cancer patients whose disease was not radically resectable.

\begin{tabular}{|c|c|c|}
\hline \multirow[b]{2}{*}{ Gender } & Male & $66,7 \%$ \\
\hline & Female & $33,3 \%$ \\
\hline \multirow{3}{*}{ Age } & Under 40 & $20,5 \%$ \\
\hline & $45-60$ & $66,7 \%$ \\
\hline & Over 65 & $13,8 \%$ \\
\hline Treatment history & Taking chemotherapy before & $50 \%$ \\
\hline \multirow{3}{*}{ Signs } & Abdominal pain & $74,4 \%$ \\
\hline & Hematochezia & $25,6 \%$ \\
\hline & Diarrhea & $23,1 \%$ \\
\hline \multirow{2}{*}{ Performance status } & PS1 & $82,1 \%$ \\
\hline & PSO & $17,9 \%$ \\
\hline \multirow{2}{*}{ Symptoms } & Bowel obstruction & $43,6 \%$ \\
\hline & Papable abdominal mass & $15,4 \%$ \\
\hline \multirow{3}{*}{ Tumor location } & Sigmoid colon & $41 \%$ \\
\hline & Hepatic flexure & $17,9 \%$ \\
\hline & Other location & $41,1 \%$ \\
\hline \multirow{4}{*}{ Distant metastatic site } & Liver metastasis & $64,1 \%$ \\
\hline & Pulmonary metastasis & $17,9 \%$ \\
\hline & Peritoneal metastasis & $25,6 \%$ \\
\hline & Retroperitoneal lymph node & $30,8 \%$ \\
\hline \multirow{2}{*}{ Number of metastasis } & $>1$ & $16.7 \%$ \\
\hline & $=1$ & $83.3 \%$ \\
\hline \multirow{2}{*}{ CEA concentration } & CEA $>20 \mathrm{ng} / \mathrm{ml}$ & 13 \\
\hline & $\mathrm{CEA} \leq 20 \mathrm{ng} / \mathrm{ml}$ & 26 \\
\hline \multirow{2}{*}{ Histopathology } & Moderately differenciated adecarcinoma & $66,7 \%$ \\
\hline & Mucinous adenocarcinoma & $33,3 \%$ \\
\hline
\end{tabular}

Table 2: Response rate of the treatment.

\begin{tabular}{|cc|}
\hline Response & $\%$ \\
\hline Completely & 0 \\
\hline Partially & 75 \\
\hline Disease stability & 16,7 \\
\hline Disease progression & 8,3 \\
\hline
\end{tabular}


respectively. The median dosage intensity of irinotecan, oxaliplatin and 5-FU calculated during the entire treatment period among 39 colon rectal cancer patients were $83.7 \%, 83.08 \%$ and $84.33 \%$, respectively.

After a median follow-up of 36 months, the median progression free survival in our study was calculated as $13.4 \pm 9$ months. The overall survival rate at $12^{\text {th }}$ month and $24^{\text {th }}$ month was estimated as $90 \%$ and $76 \%$, respectively (Figures 1 and 2).

\section{Toxic effect of FOLFOXIRI}

All patients were assessable for safety. The most common toxicitieswereneutropenia, thrombocytopenia, amenia, vomiting, diarrhea, stomatitis, hepatic toxicity, peripheral neurotoxicity. However, majority of toxic effects were at grade 1 or 2 , grade 3 or 4 was commonly seen in neutropenia. Of which, granulocytopenia of grade $1 / 2$ occurred in $25.5 \%$ of patients, while grade 3 and 4 occupied a lower rate of $14.1 \%$ and $9.3 \%$, respectively. Thrombocytopenia and anemia account for very low percentage of $3.2 \%$ and $8.3 \%$, mainly at grade 1 . Liver toxicity was the most prevalent non-hematological adverse event and account for the highest proportion of $52.5 \%$, followed by vomiting and diarrhea with the figure of $18.3 \%$ and $6.9 \%$. All of them were scored at grade $1 / 2$ (Table 3).

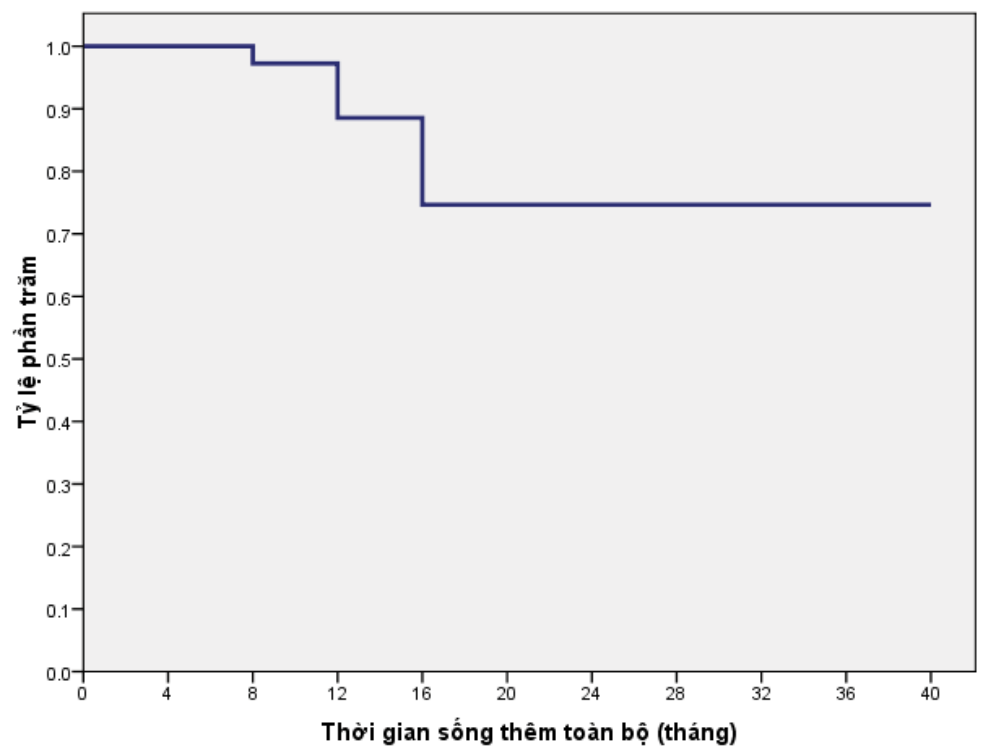

Figure 1: Progression free survival.

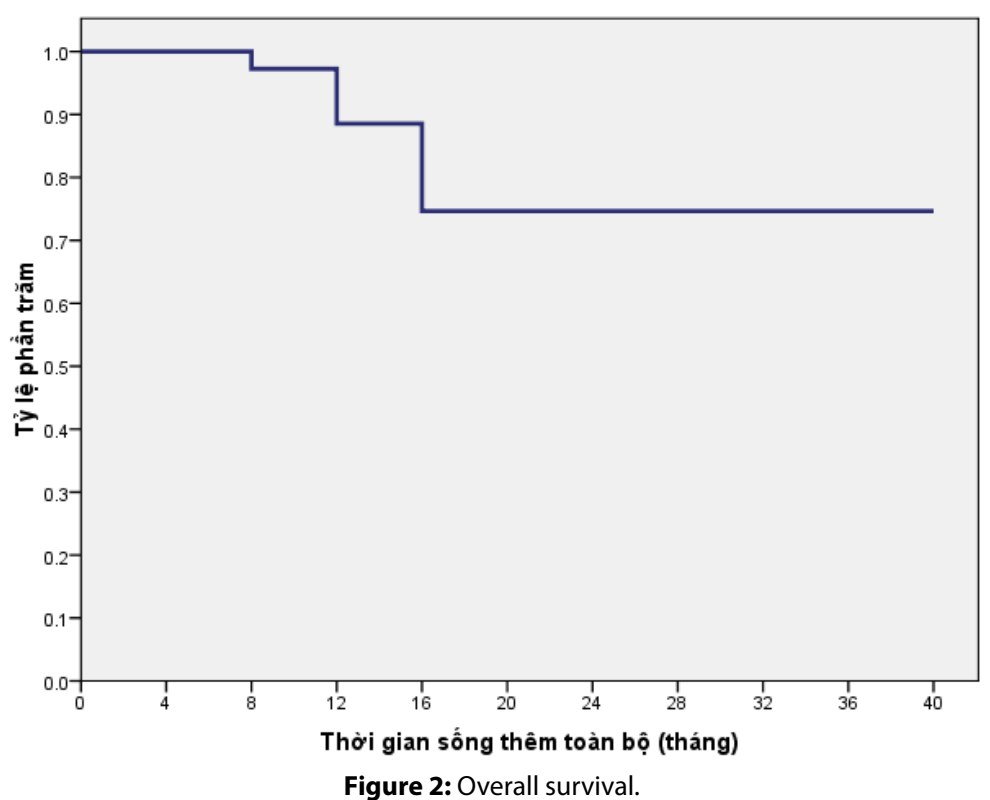


Table 3: Toxic effect of FOLFOXIRI regimen.

\begin{tabular}{|lcccc|}
\hline Adverse events & Grade $\mathbf{0}(\mathbf{\%})$ & Grade $\mathbf{1 / 2}(\mathbf{\%})$ & Grade 3/4 (\%) & Sum (\%) \\
\hline Granulopenia & 51.1 & 25.5 & 23,4 & 100 \\
\hline Thrombocytopenia & 96.8 & 3.2 & 0 & 100 \\
\hline Anemia & 91.7 & 8.3 & 0 & 100 \\
\hline Vomiting & 74.23 & 23 & 2.67 & 100 \\
\hline Diarrhea & 93.1 & 6.9 & 0 & 100 \\
\hline Oral toxicity & 96.57 & 3.63 & 0 & 100 \\
\hline Liver toxicity & 47.5 & 52.5 & 0 & 100 \\
\hline
\end{tabular}

\section{Discussion}

Over the past years, improvements in chemotherapy for treatment of patients with metastatic colorectal cancer have resulted in significant benefits in terms of antitumor activity and efficacy $[4,5]$. Several documents had showed that the best results are obtained from patients who are exposed to all three of the main active agents (5-FU, irinotecan and oxaliplatin), but that in a sequential strategy only $60 \%$ to $80 \%$ of patients are able to continue the second-line treatments and therefore to be able to exposed to all these three agents. These considerations support the strategy to develop potentially more active first-line regimens combining 5-FU with both irinotecan and oxaliplatin. Thus, we conducted the study to evaluate effectiveness of a three-drug combination of irinotecan, oxaliplatin and 5-FU/LV (FOLFOXIRI) using the treatment sequence irinotecan, oxaliplatin, 5-FU, orderly.

Falcone et al, 2002, conducted a study on 39 stage IV metastatic colorectal cancer patients by using Irinotecan $175 \mathrm{mg} / \mathrm{m} 2$, Oxaliplation $100 \mathrm{mg} / \mathrm{m}^{2}$ combined with Leucovorin $200 \mathrm{mg} / \mathrm{m}^{2}$ in day 2 and continuos infusion of $5 \mathrm{FU} 3800 \mathrm{mg} / \mathrm{m}^{2}$ on day 2 and day 3 . The result showed five $(11.9 \%)$ patients with complete response and $25(59.5 \%)$ patients with partial response, thus, overall response rate $71.9 \%$ [3].

Souglakos et al. treated 31 metastatic colorectal cancer patients with first-line irinotecan $150 \mathrm{mg} / \mathrm{m}^{2}$ on day 1 , oxaliplatin $65 \mathrm{mg} / \mathrm{m}^{2}$ on day 2 , followed by standard de Gramont schedule LV-modulated bolus infusional 5-FU on day 2 and day 3, repeated every 2 weeks, and achieved overall response rate of $58.1 \%$, in which $6.5 \%$ complete response and $51.6 \%$ partial response, rate of disease stabilization and progressive disease in $25.8 \%$ and $16.1 \%$ of patients, respectively [4].

Azmy et al., published the results of phase II studies, based on 60 patients with stage IV colorectal cancer were divided into two groups: group 1 was treated with FOLFOXIRI regimen with higher dosage of Irinotecan compared with Falcone's study $\left(180 \mathrm{mg} / \mathrm{m}^{2}\right.$ vs. $165 \mathrm{mg} /$ $\mathrm{m}^{2}$ ), and other drugs had similar doses, group 2 was treated with FOLFIRI. The overall response rate of group 1 was significantly higher than that of group 2 (60\% vs. $33 \%, \mathrm{p}=0.007)$ [5].

Falcone et al. (2007), after 5 years, published results of phase III studies performed in two groups: group 1 was treated with FOLFOXIRI at a lower dosage compared to phase II study (Irinotecan 165 $\mathrm{mg} / \mathrm{m}^{2}$, Oxaliplatin $85 \mathrm{mg} / \mathrm{m}^{2}$, $5 \mathrm{FU} 3200 \mathrm{mg} / \mathrm{m}^{2}$ and standard Leucovorin $200 \mathrm{mg} / \mathrm{m}^{2}$ ), group 2 was treated with FOLFIRI regimen. The author observed overall responde of $60 \%$, of which complete response in $8 \%$ and partial response in 58\% of patients in group 1. Compared with the corresponding proportion of $34 \%$, $6 \%$ and $35 \%$ in group 2 , the difference was statistically significant [6].

In our study population, there were no cases of complete response, 9 cases of partial response (75\%), 2 cases of disease stability (16.7\%) and 1 case of progressive disease (8.3\%). The overall response rate is among the highest reported in the literature for any chemotherapy regimen in metastatic colorectal cancer. The median dose intensities were $83.7 \%$ with Irinotecan, $83.08 \%$ with Oxaliplatin, $84.33 \%$ with $5 \mathrm{FU}$. This is the lowest dose intensity of chemotherapy compared to those four reference studies, except for Souglakos et al.'s study [2]. Regarding to patients' characteristics, based on three main factor: median age, prevalence of patients treated with chemotherapy before, severity of disease, our patient group present present an younger median age (54 years), the second lowest number of patients with metastatic sites $>1 \quad(16.7 \%$, just behind the figure of Azmy et al.'s study with 9\%), and approximately 50\% of patients who had been taken with chemotherapy previously (middle-ranking among 4 cited studies). Thus, the characteristics of our studied population are relatively more favorable than those of other groups in mentioned studies.

During a follow-up period of 40 month, the median progression free survival of this study was recorded as 
$13.37 \pm 9$ months. This result was comparable to foreign studies using the same regimen. Particularly, Falcone et al.'s study, (2002) revealed a median progression free survival of 10.4 months while Souglakos et al.' study reported the progression free survival of 13 months on average [4]. Furthermore, in comparison with other regimen, FOLFOXIRI significantly increased PFS by approximately 3 months, and it reduced the risk of early progression by half [6]. The 12th month- and 24 th month- overall survival rate were $90 \%$ and $76 \%$, respectively.

\section{Some side effects of FOLFOXIRI}

Granulocytopenia: An important disadvantage of FOLFOXIRI is increase of side-effect, but in an acceptable level. Neutropenia was the most common hematological toxic effect with proportion of $48.9 \%$. In particular, the incidence of grade $1 / 2$ granulocytopenia was $25.5 \%$ while grade $3 / 4$ was observed in $23.4 \%$ of patients. There was no case of fever caused by neutropenia. Interestingly, neutropenia was one of toxicity with highest rate in studies by foreign authors. Falcone et al.'s study suggested that $14 \%$ of patients had at least one episode of neutropenia fever [3]. Souglakos et al. found that grade 3 and grade 4 neutropenia happended in 14 patients (45\%), two patients had neutropenia fever (6\%) [4]. Compared to foreign authors, our result of neutropenia is somewhat lower. In addition to the reason for carefully selecting patients, detecting and excluding cases of drug contraindications, there still was very close monitoring process. Patients were screened for blood count routinely at 8 th day after chemotherapy. Cases with grade 2 or above were injected with leukocyte stimulants and closely observed, daily white blood cell counts was measured to administer leukocyte-stimulating drugs if necessary until blood cound reach over 2000 white blood cells/ $\mathrm{ml}$. In addition, dosage adjustment for each patient was based on level of granulocytopenia in the previous cycle of treatment. Cases with grade 3 granulocytopenia were isolated in sterile rooms and broad-spectrum antibiotic prophylaxis was used until white blood cells increased to above $2000 / \mathrm{ml}$.

Anemia and thrombocytopenia: The rates of anemia and thrombocytopenia in our study were 8.3\% and $3.2 \%$, respectively. Most of side effects of anemia $(6.5 \%)$ and thrombocytopenia were at grade 1 . The rate of grade 2 anemia was very low with $2.3 \%$ and these side effects at grade 3 and 4 did not appear in the study. Also, the percentages of anemia and thrombocytopenia of foreign studies were very low $(<10 \%)$ [4-6]. The three drugs Oxaliplatin, 5FU and Irinotecan have no effect on thrombocytopenia occurence clearly. Regarding to anemia, only oxaliplatin can cause a small erythropoietic anemia because a small fraction $(-7 \%)$ of oxaliplatin penetrate into red blood cells and breaks down the red blood cells after 48 days. Nevertheless, because only a small proportion of oxaliplatin can get into red blood cells, the levels of anemia of regimens containing oxaliplatin in general and of FOLFOXIRI in particular are not high.

Vomiting: There were 42 events of vomiting during chemotherapy. However, no incidence of vomiting at grade 4 , grade 3 occrued in $2.67 \%$ of the patients enrolled in this study. The remaining was mainly infusions causing vomiting at grade 1 (74.23\%) and grade $2(23 \%)$. The toxicity rate was somewhat lower than Falcone et al.'s study, in which the incidence of vomiting was $60 \%$ for grade $1,53 \%$ for grade 2 and $13 \%$ for grade 3 [3]. The causes of this type of vomiting are usually related to psychological factors, psychological fear will stimulate pneumogastric nerve to cause vomiting. To solve this problem, we usually take supplements of pills (Diazepam) for patients with a history of severe vomiting in the previous infusion. In addition to using the 5-HT3 class of anti-vomiting medication, we also combined two other classes in a routine way: Corticosteroids (dexamethasone) have anti-vomiting mechanism on the central nervous system; Primperan has anti-vomiting mechanism by D4 receptor blockade. Several severe vomiting cases (grade 3) were also given Haloperidol, a chemotherapyinduced P-receptor blocker in the CTZ (chemo-trigger zone).

Diarrhea: The incidence of diarrhea in our study group was $6.9 \%$. Most patients developed diarrhea at later time (after 24 hour of chemical infusion). There was no case of diarrhea in grade 3 or 4 . Our result about diarrhea rate was smaller than that of foreign authors. For example, Souglakos et al. implemented study on 31 patients with stage IV colorectal cancer with the similar regimen and found that grade 3 and 4 diarrhea was presented in 10 cases (32\%) [4]. Besides, the rate of grade 3, 4 diarrheas in Falcone et al.'s research (2007) was $20 \%$, with no significant difference from FOLFIRI group [6]. Irinotecan, in addition to be Topoimerase II enzyme inhibitor, inhibit Acetylcholinesterase, an enzyme that decomposes neurotransmitter of Acetylcholi receptor. Therefore, using Irinotecan will stimulate parasympathetic nervous system which intensifies bowel movement and results in diarrhea. All of our patients are taking Atropin to prevent this side 
effect. Depending on the severity of patient's diarrhea, Atropin is added during infusion.

Oral toxicity: $3.63 \%$ of patients with oral toxicity had been detected in this study and all were at grade 1. FOLFOXIRI regimen not using bolus 5-FU as a feature that causes low rate of stomatitis. Studies of foreign author also have a low incidence of this toxicity (below 5\%) [4,5]. Cases with oral toxicity was detected and treated early, which limit significantly the severity of this unwanted effects.

Hepatic toxicity: The most prevail non-hematological adverse event was liver toxicity, accounting for $52.5 \%$ of patients treated by FOLFOXIRI, but most of them stop at grade 1 (50\%), followed by grade 2 with only $2.5 \%$. Similarly, liver toxicity in majority of foreign studies amounted to highest percentage at grade 1 while the figure of grade 2 toxicity was very small [4-6]. Of three drugs used in this study, Oxaliplatin was metabolized and excreted mainly in kidney so there was no effect on the liver. 5FU and irinotecan are metabolised and excreted primarily in the liver, which can cause hepatitis due to the drug. However, the level of hepatitis is usually mild and self-healing after chemotherapy discontinuation. During treatment, we focus on the use of infusion after chemotherapy to improve liver function, which helps reduce liver enzymes. At the same time, patients are thoroughly consulted about the risk of increased hepatic enzyme due to drug use. All of these measures have contributed to minimizing liver toxicity in this study.

\section{Conclusion}

In conclusion, the FOLFOXIRI chemotherapy is a highly effective regimen demonstrated by an increase in response rate, progression free survival and has adverse events at manageable and well tolerated levels during first-line treatment of metastatic colon cancer patients. The regimen can be widely applied in cancer treatment facilities throughout the country, especially for patients who are still in good condition and cannot afford to take expensive target therapy.

Executive summary

Background: In recent years, the new trend in treatment of colorectal cancer is the use of target drug. We conduct the study to evaluate efficacy of triplet combination chemotherapy of 5-fluorouracil/leucovorin, oxapilatin, and irinotecan (FOLFOXIRI) in the treatment of stage IV colorectal cancer patients.

Method: Uncontrolled clinical trial carried on 39 stage IV colorectal cancer patients

Results: The overall response rate of the treatment was $75 \%$. Mean of progression free survival was $13.4 \pm 9$ months. The overall survival rate at $12^{\text {th }}$ month and $24^{\text {th }}$ month were $90 \%$ and $76 \%$, respectively. The proportion of granulocytopenia was $48.9 \%$, no grade 3 or 4 . Side effect beyond hematology was most seen in hepatic toxicity with $52.5 \%$, mainly at grade 1 . Vomiting was $18.3 \%$, all at grade 1 . Other toxicity was very low at percentage.

Conclusion: The triplet combination FOLFOXIRIN chemotherapy improves the outcome of patient with metal colorectal cancer in term of response rate, overall survival rate and progression free survival, and the level of toxicity was acceptable.

\section{References}

1. Agabegi ED, Agabegi SS. Step-Up to Medicine (Step-Up Series). Lippincott Williams \& Wilkins. 107: 3-1 (2008).

2. Grothey A, Sargent D, Goldberg RM, Goldberg RM, Schmoll HJ. Survival of patients with advanced colorectal cancer improves with the availability of fluorouracil, leucovorin, irinotecan and oxaliplatin in the course of treatment. J Clin Oncol. 22: 12091214 (2004).

3. Falcone A, Masi G, Allegrini G, et al. Biweekly chemotherapy with oxaliplatin, irinotecan, infusional fluorouracil and leucovorin: a pilot study in patients with metastatic colorectal cancer. J Clin Oncol. 20: 4006-4014 (2002).

4. Souglakos J, Mavroudis D, Kakolyris S, et al. A triplet combination with irinotecan (cpt-11) plus oxaliplatin (l-ohp) plus continuous infusion 5-fluorouracil (5 FU) and leucovorin (LV) as first-line treatment in metastatic colorectal cancer (MCC): a multicenter phase II trial. J Clin Oncol. 20: 26612667 (2002).

5. Azmy MA, Nasr KE, Gobran NS, Mostafa MY. Infusional fluorouracil, leucovorin, oxaliplatin and irinotecan (FOLFOXIRI) compared with infusional fluorouracil, leucovorin and irinotecan (FOLFIRI) as first-line treatment for metastatic colorectal cancer. J Cell Sci Ther. 3: 125 (2012).

6. Falcone A, Ricci S, Brunetti I, et al. Phase III trial of infusional fluorouracil, leucovorin, oxaliplatin, and irinotecan (FOLFOXIRI) compared with infusional fluorouracil, leucovorin, and irinotecan (FOLFIRI) as first-line treatment for metastatic colorectal cancer: the Gruppo Oncologico Nord Ovest. J Clin Oncol. 25: 1670-1676 (2007). 